\title{
CARACTERIZAÇÃO MAGNÉTICA DE LÂMINAS DE AÇO SILÍCIO E AVALIAÇÃO DAS PERDAS NO FERRO SOB VÁRIOS REGIMES DE INDUÇÃO
}

\author{
Nelson J. Batistela* \\ jhoe@grucad.ufsc.br \\ Renato Carlson* \\ rcarlson@ieee.org
}

\author{
Nelson Sadowski* \\ nelson@grucad.ufsc.br \\ Jean V. Leite* \\ jean@grucad.ufsc.br
}

*Grucad, Depto. de Eng. Elétrica, Universidade Federal de Santa Catarina, P. 0. Box 476, 88049-900

Florianópolis, SC, BRASIL

\begin{abstract}
The iron losses are evaluated, measured and analyzed. The Si-Fe sheets are magnetically characterized under a sinusoidal induction waveform and at one frequency. In this study, the iron losses are predicted with the magnetic core working in a non-sinusoidal regime and other frequencies and we present a novel magnetic material characterization methodology in function of the flux density variation in the material, trying to denote some problems in the characterization analysis and to explore the iron losses under magnetic flux regimes with sinusoidal and non-sinusoidal waveforms.
\end{abstract}

KEYWORDS: Iron losses separation, magnetic characterization, hysteresis losses measurement, pulse waveform excitation.

\section{RESUMO}

Este estudo se refere a caracterização, medição e análise das perdas no ferro. Apresenta-se uma metodologia de caracterização dos materiais magnéticos em função da variação da indução no material, procurando apontar, na medida do possível, algumas questões que existem

Artigo submetido em 20/12/00

1a. Revisão em 29/05/01

Aceito sob recomendação do Ed. Assoc. Prof. Edson H. Watanabe nestes ensaios e explorar as perdas no ferro sob regimes de fluxo magnético nas formas senoidal e não-senoidal, em função da variação da freqüência de operação.

PALAVRAS-CHAVE: Separação das perdas no ferro, caracterização magnética, medida da perda por histerese, perdas no ferro sob regimes não-senoidais.

\section{INTRODUÇÃO}

Os materiais ferromagnéticos exercem um papel importante na confecção de um dispositivo eletromagnético, pois são os responsáveis pela ordenação das linhas de fluxo no circuito magnético e têm uma importância na medida que os mesmos podem determinar a eficiência, o custo e o tamanho dos dispositivos. Os usuários de lâminas de aço silício percebem um aumento sensível das perdas magnéticas em relação às perdas especificadas nos catálogos dos fabricantes, obtidas conforme os métodos NBR 5161, JIS 2550/86, Din 50462, ASTM A34 (Acesita). Além disso, cada método fornece valores diferentes de perdas. A deterioração do desempenho das mesmas foi inicialmente atribuída somente à geometria do circuito magnético, ao corte das lâminas, à prensagem mecânica e à anisotropia magnética. Na evolução dos estudos, percebeu-se a existência de outras formas de perdas além das conhecidas até então. Aparecem valores medidos superiores ao esperado, que foram chama- 
dos de "perdas por excesso" (Berttoti, 1983, 1984, 1985, 1988).

Os circuitos magnéticos eram percorridos por um fluxo de forma geral senoidal, pois um dispositivo eletromagnético era projetado para operar na faixa linear da curva B-H, garantindo que o valor da indução magnética estivesse bem aquém da região chamada "joelho" (por isso os motores antigos eram mais robustos e volumosos). Hoje, os aparelhos são projetados e otimizados para operarem o mais próximo possível do joelho da curva de magnetização, onde acaba a relação linear entre fluxo magnético e sua força magnetomotriz para criá-lo. Mesmo com uma alimentação puramente senoidal, em razão da especificidade do circuito magnético, o fluxo local pode ser distorcido em algumas regiões de um circuito magnético de uma máquina elétrica (Basak et alii, 1982). Isto é causado pela saturação local provocada pela não homogeneidade do caminho magnético (por exemplo nas máquinas girantes há o efeito provocado pelas ranhuras) e pela variação da anisotropia magnética (Moses et alii, 1989). Nestas regiões, as perdas no ferro são, em geral, maiores que a média das perdas no resto do circuito. Além disso, com a introdução da alimentação por meio de conversores estáticos, a forma de onda do fluxo passou a ser diferente da senoidal (Allano et alii, 1987). Esses regimes têm conseqüências sobre o desempenho das lâminas: as perdas no ferro aumentam geralmente em proporções mais ou menos importantes, dependendo da taxa de distorção do fluxo magnético. A meta perseguida pelos construtores de ferramentas destinadas ao projeto de dispositivos eletromagnéticos é modelar os fenômenos magnéticos nos materiais e conhecer a distribuição local do fluxo (Ferreira da Luz et alii, 2000).

Até o passado recente, a abordagem física sobre as perdas no ferro chegou a um formalismo microscópico muito complexo e de difícil utilização pelo engenheiro. A escola italiana de eletromagnetismo, o "Instituto Elettrotecnico Nazionale Galileo Ferraris" de Torino, desenvolveu uma teoria física da quantificação e separação das perdas nas lâminas magnéticas, possuindo a vantagem de deduzir, dos parâmetros microscópicos, uma modelagem macroscópica global sob regime de indução na forma senoidal. A presente abordagem do material se apoia sobre esta modelagem matemática do fenômeno físico. A vantagem da análise física é a compreensão do mecanismo de geração das perdas durante o processo de magnetização do material. O método de separação das perdas (Fiorillo et alii, 1990) explorado neste artigo e o método de predição das perdas formulado por Amar e Protat (1994) carecem ainda de um modelo satisfatório para a perda por histerese, como por exemplo o de Jiles-Atherton (Hernandez et alii, 2000).

\section{EQUAÇÕES DE ESTIMAÇÃO DAS PERDAS NO FERRO}

As perdas totais no ferro $\mathrm{W}_{t o t}$, por período e por unidade de massa, em lâminas de aço silício submetidas a uma indução periódica alternada são dadas pela soma das perdas por histerese $\mathrm{W}_{h}$, por correntes de Foucault calculadas pelo modelo clássico $\mathrm{W}_{f}$ e por excesso $\mathrm{W}_{e}$. De acordo com o estudo realizado por Bertotti e Fiorillo, elas são obtidas através da equação (1), onde f é a freqüência de operação, de período $\mathrm{T}$, com lâminas de espessura d, condutividade elétrica $\sigma$ e densidade específica $\mathrm{m}_{v}$. S é a seção magnética efetiva. A equação (1) é função da variação da indução no tempo dB/dt, e válida para qualquer forma de onda.

$$
\begin{aligned}
W(t)_{t o t}= & W(t)_{h}+\frac{\sigma d^{2}}{12 f m_{v}} \frac{1}{T} \int_{0}^{T}\left(\frac{d B(t)}{d t}\right)^{2} d t+ \\
& +\frac{\sqrt{\sigma G V_{o} S}}{f m_{v}} \frac{1}{T} \int_{0}^{T}\left|\frac{d B(t)}{d t}\right|^{1,5} d t[\mathrm{~J} / \mathrm{Kg}]
\end{aligned}
$$

O segundo termo aditivo da equação (1) representa as perdas por correntes de Foucault $\mathrm{W}_{f}$, as quais provêm das correntes induzidas no núcleo quando percorrido por um fluxo variável. A perda por histerese, equação (2), corresponde ao valor da área da curva de histerese e dos seus laços menores, se existirem. O emprego e a solução da equação (2) não é tão fácil. Um dos métodos mais utilizados no cálculo deste tipo de perda é a equação empírica (3) encontrada por Steinmetz em 1892. A constante $\mathrm{k}_{h}$ depende do material e do sistema de unidades utilizado, e o expoente $\alpha$ depende apenas do material. No processo de caracterização magnética do material apresentado neste artigo, utiliza-se o modelo de Steinmetz da evolução da perda por histerese em função da indução máxima.

$$
\begin{gathered}
W_{h}=\frac{1}{m_{v}} \int_{\left.B\right|_{0}}^{\left.B\right|_{T}} H(t) d B(t)[\mathrm{J} / \mathrm{Kg}] \\
W_{h}=k_{h}\left(B_{m}\right)^{\alpha}[\mathrm{J} / \mathrm{Kg}]
\end{gathered}
$$

Pesquisadores constataram que a perda por histerese medida obedece à fórmula de Steinmetz até certos valores de indução máxima $\mathrm{B}_{m}$. Landgraf et alii (1999, 2000) propõe separar as perdas de histerese em dois componentes: em "baixas induções" e em "altas induções". Richter (Liwschitz) propõe a equação (4) para o cálculo da perda por histerese $\mathrm{P}_{h}$. Os coeficientes "a" e "b" são constantes dependentes do material. O resultado 
apresentado na figura 6 também confirma a mudança da tendência da perda por histerese nas altas induções.

$$
P_{h}=a f\left(B_{m}\right)+b f\left(B_{m}\right)^{2}[\mathrm{~W} / \mathrm{Kg}]
$$

A evolução das perdas por histerese conduz a algumas reflexões: a) por definição, a perda por histerese é delimitada em seu valor máximo pela curva de histerese, mostrada na figura 1. Na região de saturação ao se aumentar a indução, a área correspondente a este aumento da indução B e de sua correspondente intensidade de campo H não é tão significativa. Assim, a uma dada indução B, a evolução da perda por histerese deveria apresentar uma certa saturação. b) A fórmula de Steinmetz tem seu expoente variando para lâminas de aço silício entre os valores 1,4 a 1,8. Ora, a tendência dada por este modelo é sempre crescer "ad infinitum" de maneira exponencial. Algumas questões podem ser levantadas: os ensaios estão medindo realmente só a perda por histerese? Os modelos são adquados? É um problema oriundo de outros fenômenos que foram negligenciados (mudança do caminho magnético médio, as juntas no quadro de Epstein, direção e corte das lâminas,...)? Ou é o efeito de um outro fenômeno de perda ainda não discutido pela comunidade técnico-científica?

No processo de magnetização, o fenômeno de histerese é provocado por dois mecanismos conhecidos: movimentação das paredes e rotação de domínios. Na região de deslocamento das paredes de domínio, a energia utilizada no processo é dissipada, enquanto na rotação seria conservada. Mas mesmo onde predomina a magnetização por rotação ocorre energia dissipada, pois o ramo descendente não volta sobre o ramo ascendente da curva de histerese. Esta perda pode ser devida a uma parcela de energia dissipada em rotações irreversíveis, e/ou à movimentação de paredes de domínio durante a aniquilação de domínios (ramo ascendente) e/ou durante a nucleação de domínios (ramo descendente).

A figura 1 mostra medidas sob tensão senoidal no secundário do quadro de Epstein à $1 \mathrm{~Hz}$. A permeabilidade relativa $\mu_{r}$ em função de $\mathrm{H}$, obtida da curva de magnetização inicial, está apresentada na figura 1 ( $\mu$ e $\mu_{o}$ são as permeabilidades magnéticas do material e do vácuo, respectivamente).

O terceiro termo aditivo da equação (1) é a "perda por excesso". A sua suposta origem é decorrência do excesso de correntes induzidas (além das calculadas pelo modelo clássico) devido ao fenômeno de deslocamento das paredes dos domínios magnéticos. Bertotti abordou de uma forma estatística o processo de magnetização dinâmica e as perdas por excesso. Ele definiu uma entidade física, o "objeto magnético" $\mathbf{O M}$, pela qual a magnetização se inverte. A noção dos OMs se justifica pelo fato de que o deslocamento de uma parede de um domínio, ou segmento de parede, a qual é a origem da variação da magnetização no material, não pode ser feito de forma isolada. O deslocamento de uma parede pode provocar um deslocamento de outros segmentos da mesma parede e de várias. Neste caso, diz-se que os diferentes segmentos de uma mesma parede ou conjunto delas são correlacionados. Supõe-se que inicialmente apenas um $\mathbf{O M}$ esteja ativo. Quando $\mathrm{dB}(\mathrm{t}) / \mathrm{dt}$ cresce, um campo de excesso $\mathrm{H}_{e}(\mathrm{t})$ é necessário para contrabalançar a frenagem aumentada pelas correntes induzidas em excesso. Uma vez que ele seja suficientemente grande, este campo exerce sobre os outros OMs uma pressão para torná-los ativos, os quais vão agir da mesma maneira sobre os outros OMs passivos, progressivamente aumentando o número de OMs ativos e tornando a magnetização homogênea. A experiência mostra que a relação entre o número de OMs ativos e o campo $\mathrm{H}_{e}(\mathrm{t})$ é linear em numerosos materiais cristalinos, e também no Fe-Si (Bertotti, 1983). Esta relação é traduzida pelo parâmetro $\mathrm{V}_{o}$, eqüivalente a um campo coercitivo e caracteriza a oposição dos $\mathbf{O M s}$ em se ativar. A determinação do parâmetro $V_{o}$ necessita da medida do número de OMs ativos e do campo $\mathrm{H}_{e}(\mathrm{t})$. G é uma constante que representa o coeficiente de atrito de um OM. A determinação destes parâmetros exige alta tecnologia e conhecimento adequado para medi-los. No meio industrial como no acadêmico na área da engenharia elétrica, a determinação dos parâmetros referente às características micro-estruturais é evitada ao máximo. A equação (1) apresenta a primeira formulação física das perdas por excesso, sendo coerente com conclusões de outros pesquisadores (Haller et alii, 1970).

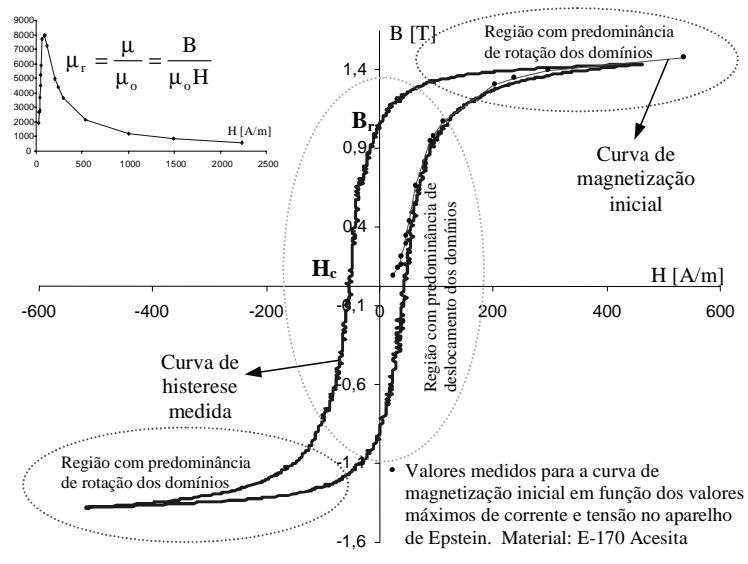

Figura 1: Curva representativa da histerese medida à $1 \mathrm{~Hz}$.

As equações (1) e (2) mostram que a variação da indução magnética no material ao longo do tempo é o que provoca as perdas, sendo um fato coerente com a natu- 
reza do processo de magnetização do material. Tendo em vista a necessidade do controle da indução magnética no material, isto é, não existir distorções (harmônicos) na forma do fluxo, obriga-se que a forma de onda da indução seja senoidal. Isto proporciona uma maneira simples de separar as perdas no ferro. Nos ensaios de caracterização, esta exigência é atendida pelo modo de se alimentar o dispositivo que aloja as amostras (Batistela et alii, 2000).

Para uma forma de onda senoidal, a perda total $\mathrm{W}_{t o t}^{s}$ em uma freqüência base $f_{o}$ é dada pela equação (5) em função da indução máxima $\mathrm{B}_{m}$. Pode-se escrever a equação (5) como a equação (6) em função das constantes $\mathrm{k}_{h}$, $\alpha, \mathrm{k}_{f}$ e $\mathrm{k}_{e}$. Na caracterização experimental, determinase estas quatro constantes. A perda pelo fenômeno de histerese é invariável com a freqüência. Entretanto, as constantes das perdas dinâmicas, $\mathrm{k}_{f}$ e $\mathrm{k}_{e}$, podem ser corrigidas para uma outra freqüência $f$ diferente da freqüência base $\mathrm{f}_{o}$, conforme a equação (7) (Fiorillo e Novikov, 1990).

$$
\begin{gathered}
W_{t o t}^{s}=k_{h} \mathbf{B}_{m}^{\alpha}+\frac{\sigma(\pi d)^{2}}{6 m_{v}} f_{o} \mathbf{B}_{m}^{2}+ \\
\frac{8,763}{m_{v}} \sqrt{\sigma G V_{o} S} \sqrt{f_{o}} \mathbf{B}_{m}^{1,5} \quad[\mathrm{~J} / \mathrm{Kg}] \\
W_{t o t}^{s}=k_{h} \mathbf{B}_{m}^{\alpha}+k_{f} \mathbf{B}_{m}^{2}+k_{e} \mathbf{B}_{m}^{1,5}[\mathrm{~J} / \mathrm{kg}] \\
\left.k_{f}\right|_{f}=\left.\frac{f}{f_{o}} k_{f}\right|_{f_{o}} \text { e }\left.k_{e}\right|_{f}=\left.\sqrt{\frac{f}{f_{o}}} k_{e}\right|_{f_{o}}
\end{gathered}
$$

Amar e Protat (1994) propõem um modelo de previsão analítica da evolução das perdas para regimes de tensão para formas de onda pulsadas, incluindo a do tipo PWM a três níveis. Baseiam-se nos trabalhos de Bertotti, de Fiorillo (intervalo de tempo de subida $\tau$ da indução na forma trapezoidal) e de Nakata (1989) (coeficiente do fator de forma $F_{c}$ ). Utilizando a idéia sobre o coeficiente $\mathrm{F}_{c}$, eles formulam uma previsão da evolução das perdas para os regimes com tensões pulsadas, dada pela equação (10), a qual provém da equação (1). O coeficiente de fator de forma $\mathrm{F}_{c}$ é definido pela relação entre os fatores de forma $\mathrm{F}$ (equação (8)) das ondas distorcidas pela senoidal, de frequiências f. O parâmetro $\tau$ é a duração do impulso para forma de ondas retangulares e $\tau_{i}$ é o somatório das larguras até o iésimo impulso, em meio período, para as do tipo PWM.

$$
F=\frac{\sqrt{\frac{1}{T} \int_{t_{o}}^{t_{o}+T} v^{2}(t) d t}}{\left|\frac{2}{T} \int_{t_{o}}^{t_{o}+T / 2} v(t) d t\right|}
$$

$$
\begin{aligned}
& F_{c}=\frac{F_{r}}{F_{s}}=\frac{2}{\pi} \frac{1}{\sqrt{f}} \frac{1}{\sqrt{\tau}} \mathrm{e} \\
& F_{c}=\frac{F_{P M W}}{F_{s}}=\frac{2 \sqrt{2}}{\pi} \sqrt{\frac{1}{2 f \sum_{1}^{n} \tau_{i}}} \\
& W_{t o t}=k_{h} B_{m}^{\alpha}+\left(F_{c}\right)^{2}\left(k_{f}\right) B_{m}^{2}\left(\frac{f}{f_{o}}\right)+ \\
& +F_{c}\left(k_{e}\right) B_{m}^{1,5}\left(\sqrt{\frac{f}{f_{o}}}\right)[\mathrm{J} / \mathrm{Kg}]
\end{aligned}
$$

\section{METODOLOGIA PROPOSTA PARA A SEPARAÇÃO DOS TIPOS DE PERDA NO MATERIAL}

Neste trabalho, levanta-se os coeficientes relativos a cada tipo de perda da equação (6), em uma freqüência base $\mathrm{f}_{o}$, em função da variação da indução máxima $\mathrm{B}_{m}$ no regime senoidal puro. A metodologia aqui proposta de separação das perdas como curvas em função da indução máxima obriga que os coeficientes de cada tipo de perda e os expoentes da indução máxima sejam conforme o modelo dado pela equação (6), exemplificados na caracterização da figura 6 . Conforme a modelagem teórica, estes coeficientes e expoentes devem ser constantes para toda a variação da indução em um material. A curva da perda por histerese $\mathrm{W}_{h}^{s}$ é medida em uma freqüência baixíssima, chamada de operação quase estática $\left(\mathrm{f}<<\mathrm{f}_{o}\right.$ ). Na figura 2, constata-se que a energia perdida em uma amostra do material A, para freqüências menores que $1 \mathrm{~Hz}$ tende ao valor nulo. Isso parece ser coerente, pois no regime contínuo não existe perda pelo fenômeno de histerese. Porém para uma amostra $\mathrm{B}$, este fenômeno não foi constatado, vide figura 2. Assim, julga-se necessário fazer uma varredura da perda nas baixas freqüências para cada amostra a ser caracterizada e que avaliar a perda por histerese através do método tradicional de prolongamento da tendência das perdas para um regime estático $(\mathrm{f}=0[\mathrm{~Hz}])$ pode levar a erros. Nos ensaios apresentados foi utilizado o material A e a frequiência escolhida foi de $1 \mathrm{~Hz}$.

A curva da perda por correntes de Foucault $\mathrm{W}_{f}^{s}$ em função de $\mathrm{B}_{m}$ é calculada em uma freqüência base bem maior que a freqüência utilizada na medida da perda por histerese. Mede-se a perda total $\mathrm{W}_{\text {tot }}^{s}$, na mesma freqüência base, variando a amplitude da indução. Pelo balanço energético se obtém a curva da perda excedente $\mathrm{W}_{e}^{s}$, dada pela equação (11).

$$
W_{e}\left(B_{m}\right)=W_{t o t}^{s}\left(B_{m}\right)-\left(W_{h}^{s}\left(B_{m}\right)+W_{f}^{s}\left(B_{m}\right)\right)
$$




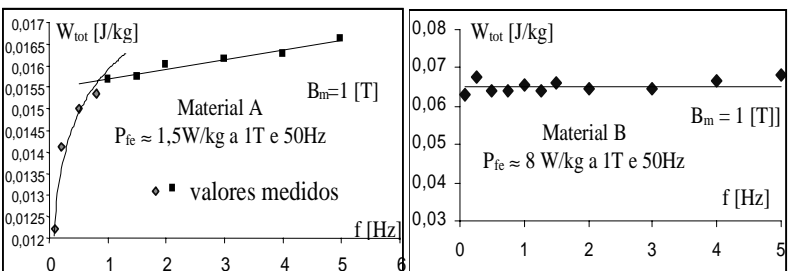

Figura 2: Energia dissipada no ferro em baixas frequiências para dois materiais.

\section{BANCADA PROTÓTIPO DE ENSAIO: CARACTERIZAČ̃̃O DO MATERIAL E DETERMINAÇÃO DAS PERDAS}

Desenvolveu-se uma bancada protótipo de ensaios para a determinação das perdas conforme a metodologia apresentada. Além da dificuldade inerente ao processo de medida, há o grande desafio de separar com satisfatória precisão os três tipos de perdas e/ou os seus efeitos, bem como alimentar adequadamente o quadro de Epstein (tipo padrão $25 \mathrm{~cm}$, Yokogawa, com 700 espiras para os enrolamentos primário $\mathrm{N}_{p}$ e secundário $\mathrm{N}_{s}$, tendo um caminho magnético médio $l_{m}$ de $0,94 \mathrm{~m}$ e uma resistência da bobina primária $R_{c u}$ de $0,691 \Omega$ ), onde são alojadas as amostras de aço silício formando o núcleo magnético. No processo de caracterização, a forma de onda do fluxo precisa ter a forma senoidal, necessitando-se impor esta forma de onda na tensão induzida no secundário. A alimentação é realizada por um inversor de onda senoidal variável em amplitude e freqüência, possível também de operar com um conteúdo harmônico ou com formas de onda pulsadas. Na literatura, a maneira utilizada para o acionamento é feita através de amplificadores lineares de áudio, os quais possuem uma banda passante de $20 \mathrm{~Hz}$ a $20 \mathrm{kHz}$. Para frequiências inferiores à $20 \mathrm{~Hz}$, ele precisa ser adaptado. Para medir as grandezas elétricas e gerar os sinais de referência, utiliza-se o software LabView da National Instruments residente em um microcomputador comunicável com um osciloscópio 2430A da Tektronix. Mede-se simultaneamente tensão e corrente nos dois canais do osciloscópio. O sinal de referência é gerado por uma placa PCI-6110E da National Instruments. A determinação das perdas nas lâminas e das demais grandezas é realizada por cálculo numérico no ambiente do software LabView em função da corrente e tensão medidas, válida para formas de ondas com conteúdo harmônico (os instrumentos convencionais não são adequados a estes ensaios, pois a corrente facilmente não tem a forma senoidal). A corrente no primário é a imagem do campo H. A integral da tensão no secundário é proporcional à indução $\mathrm{B}$. As grandezas $\mathrm{B}$ e $\mathrm{H}$ são obtidas por meio de um "instrumento virtual" no ambiente
LabView, respectivamente calculadas pelas relações (12) e (13). A integração é realizada pela equação (14), onde $\mathrm{n}$ é o número de pontos, $\Delta$ t é o intervalo de tempo entre dois pontos e $\mathrm{y}_{i}$ é o valor pontual integrado do vetor $\mathrm{X}$ contendo os pontos medidos em função do tempo.

$$
\begin{gathered}
B(t)=\frac{1}{N_{s} S} \int v_{s}(t) d t \quad[\mathrm{~T}] \\
H(t)=\frac{N_{p}}{l_{m}} i_{p}(t) \quad[\mathrm{A} / \mathrm{m}] \\
F(t)=\int f(t) d t \rightarrow y_{i}=\frac{1}{6} \sum_{j=0}^{i=0,1,2, \ldots, n-1}\left(x_{j-1}+4 x_{j}+x_{j+1}\right) \Delta t
\end{gathered}
$$

Para atingir as metas adequadas à alimentação, seguir a referência e ter a evolução livre da corrente no primário, o inversor de tensão monofásico em ponte completa, comutando à $30 \mathrm{kHz}$, com um filtro LC em sua saída, vide figura 3, possui uma das malha de realimentação com o Controle por Modo Deslizante (Sliding Mode Control - SMC) (Batistela e Perin, 1995). Havendo energia suficiente no sistema, juntamente com nenhum controle pragmático da corrente (como é no modo tradicional do SMC), o controle impõe indiretamente a força magnetomotriz para manter a tensão desejada no secundário. Esta estratégia de controle torna a saída do inversor com baixa impedância, além de dar robustez ao sistema. Pelo lado da planta, o filtro LC e as impedâncias parasitas envolvidas limitam a evolução rápida da corrente drenada $\mathrm{i}_{p}(\mathrm{t})$ da fonte de tensão contínua E. Intuitivamente, o capacitor do filtro $\mathrm{C}_{o}$ tem um valor elevado para armazenar energia, principalmente na operação em baixas frequiências, e o indutor de filtro $\mathrm{L}_{o}$ possui o valor menor possível para não dificultar a evolução da corrente. A estratégia do SMC utilizada por Batistela e Perin (1995) gera resultados satisfatórios em termos da amplitude para um ponto de operação fixo. Porém, variando as amplitudes do sinal de referência, aparece um erro. Ora, nos ensaios de caracterização e de determinação das perdas no ferro, necessita-se variar a amplitude do sinal de referência para impor os valores de $\mathrm{B}_{m}$. Assim, acrescenta-se na malha do controle da tensão no secundário um compensador do tipo PI (Proporcional e Integral), sendo seu pólo sintonizado em uma freqüência superior à do filtro LC do inversor. Há um inconveniente: como o nível contínuo não é transferido para o secundário, necessita-se de uma malha para compensar o nível de tensão contínua na entrada. Do contrário, como há um curto circuito magnético, facilmente se está na região de saturação do material. Esta malha é implementada por um compensador com duplo integrador. $\mathrm{O}$ 
esquema funcional do sistema está apresentado na figura 3.

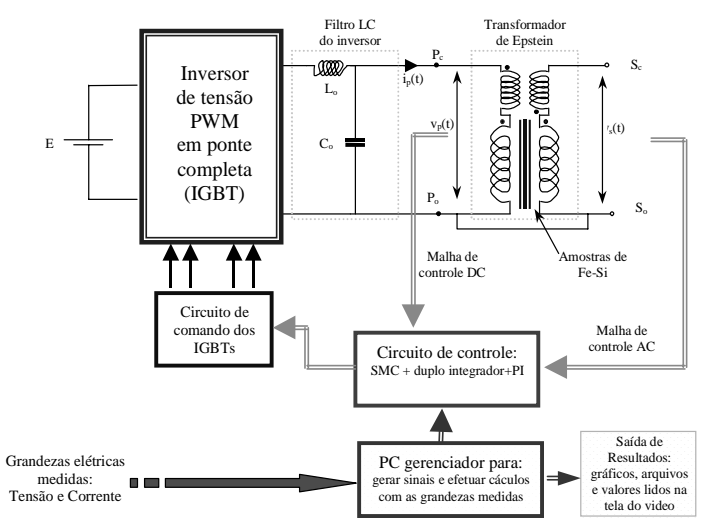

Figura 3: Esquema funcional da bancada protótipo de ensaios.

Errard (1993) utiliza a maneira expressa na equação (15) para a determinação da perda por histerese. A medida da corrente no primário é realizada através de um resistor padrão $\mathrm{R}_{\text {shunt }}\left(\mathrm{v}_{R}=\mathrm{R}_{\text {shunt }} \mathrm{i}_{p}\right)$. É o método utilizado normalmente, e provém da definição (2). A equação (15) apresenta um inconveniente: a corrente medida no primário contém parcelas de perda no resistor "shunt" e na resistência do enrolamento primário. Para solucionar este problema, optou-se por medir simultaneamente a tensão e a corrente no primário do quadro de Epstein, obtendo-se a evolução da tensão e da corrente em um período. Calcula-se a potência fornecida no tempo ao aparelho de Epstein em um período. Subtrai-se da curva da potência aparente consumida a parcela de perda no resistor equivalente à resistência elétrica do primário, restando uma curva de potência aparente magnética, a qual contém uma parcela de energia magnética armazenada e uma parcela dissipada nas amostras de Fe-Si. O processo de determinação da perda no ferro é mostrado na figura 4.

$$
W_{h}=\frac{N_{p} K_{\text {cor }}}{N_{s} m_{v}}\left(\frac{1}{T} \int_{0}^{T} v_{R}(t) v_{s}(t) d t\right) \quad[\mathrm{W} / \mathrm{Kg}]
$$

A figura 5 mostra a diferença entre os dois métodos na medida da perda pelo fenômeno de histerese em função da variação de $\mathrm{B}_{m}$, à $1 \mathrm{~Hz}$. A medição da corrente é feita através de uma ponteira de corrente. A comparação entre os resultados obtidos com o método proposto neste trabalho e aquele com o método de Errard (não descontando o efeito instantâneo de $\mathrm{R}_{c u}=0,691 \Omega$ ), mostra que a perda medida nesta amostra pelo método de Errard

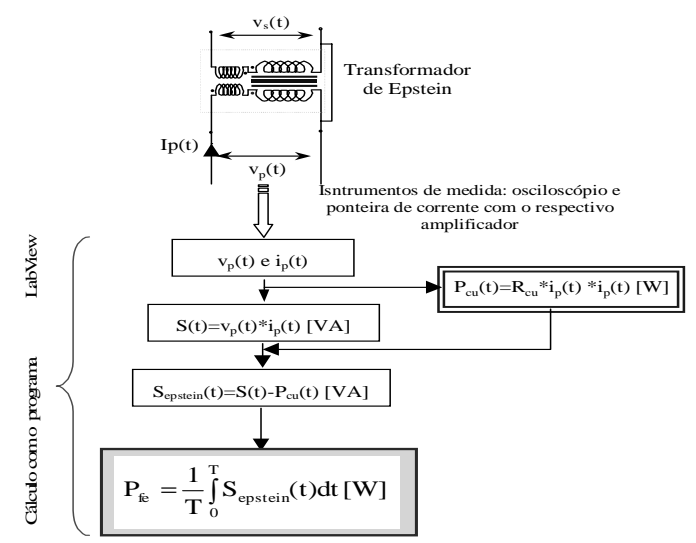

Figura 4: O processo de medida da perda no ferro.

é superior. Observa-se que na saturação e na região de baixa indução o erro relativo tende a aumentar. Provavelmente, o aumento na região de saturação se deve ao aumento da potência dissipada no cobre ocasionado pelo crescimento da corrente na saturação.

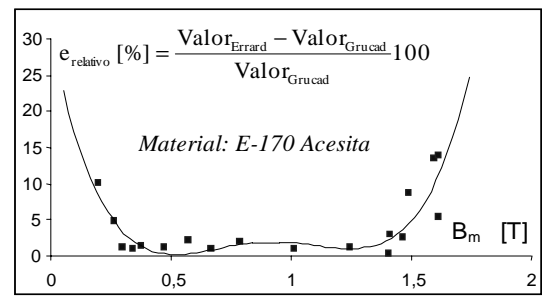

Figura 5: Diferença relativa entre os métodos à $1 \mathrm{~Hz}$.

\section{RESULTADOS}

A caracterização de uma amostra de lâminas, não tratadas, 466-50TP (especificação conforme a ABNT NBR 9025) ou E-170 0,5mm Acesita, está mostrada na figura 6 (os resultados experimentais são obtidos todos com o material A). Para induções máximas maiores que 1,4T (região de saturação), o modelo e a experimentação começam a divergir, confirmando o discorrido (Landgraf et alii,1999,2000; Liwschitz).

A figura 7 mostra o ensaio realizado a $1,2 \mathrm{~T}$ utilizando a metodologia das equações (6) e (7), ou a equação (10). Pode-se avaliar o comportamento de cada tipo de perda em função da variação da freqüência. Por exemplo, acima de $130 \mathrm{~Hz}$, a perda por correntes de Foucault passa a ser maior que a perda por histerese, para esta amostra.

Mantendo o $\mathrm{F}_{c}$ na unidade e com $\mathrm{B}_{m}$ em $0,8 \mathrm{~T}$, realizouse os ensaios para formas de onda de tensão senoidal 


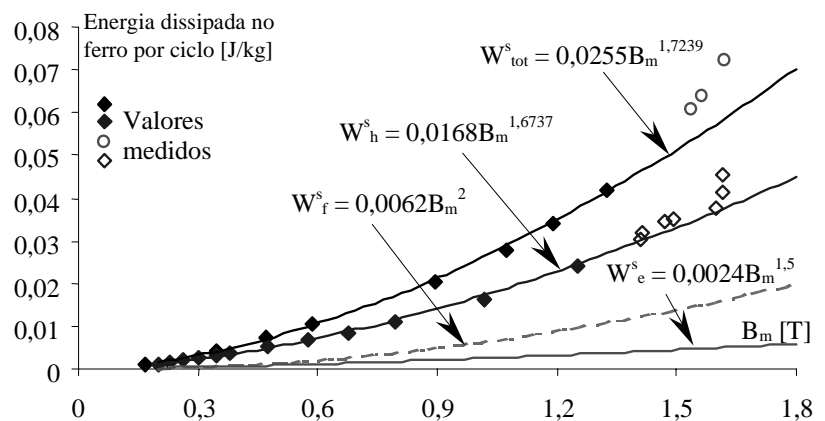

Figura 6: Caracterização do ferro: energia dissipada por ciclo $[\mathrm{J} / \mathrm{Kg}]$ total e de suas componentes para $\mathrm{f}_{0}=50 \mathrm{~Hz}$.

(figura 8), quadrada (figura 8), retangular (figura 9) e PWM a três níveis (figura 13). A figura 10 apresenta

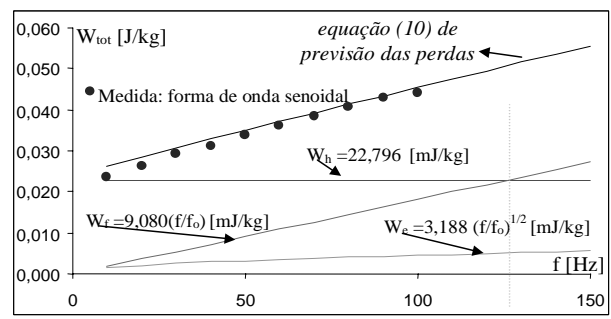

Figura 7: Energia dissipada para $\mathrm{B}_{m}=1,2 \mathrm{~T}$ para uma forma de onda de indução senoidal.

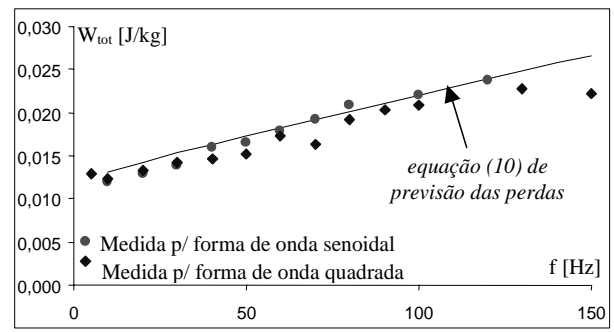

Figura 8: Energia dissipada no ferro. (Formas de onda de tensão no secundário senoidal e quadrada, $\mathrm{B}_{m}=0,8 \mathrm{~T}$ e $\mathrm{F}_{c}=1$ ).

a forma de onda de tensão retangular induzida no secundário do Epstein, para $\mathrm{f}=10 \mathrm{~Hz}$. Na figura 11, está apresentada a forma de onda de $\mathrm{H}$, imagem de $\mathrm{i}_{p}(\mathrm{t})$, em função do número de pontos adquiridos, para a mesma forma e ponto de operação. A tensão PWM à três níveis induzida no secundário do Epstein, para uma freqüência fundamental de $10 \mathrm{~Hz}$ com 30 pulsos por período, está mostrada na figura 12 .

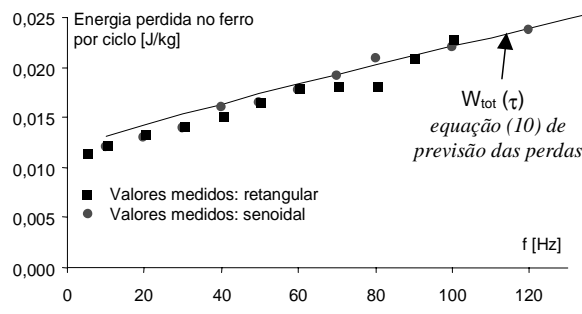

Figura 9: Energia dissipada no ferro. (Formas de onda de tensão no secundário do quadro de Epstein senoidal e retangular $\mathrm{B}_{m}=0,8 \mathrm{~T}$ e $\mathrm{F}_{c}=1$ ).

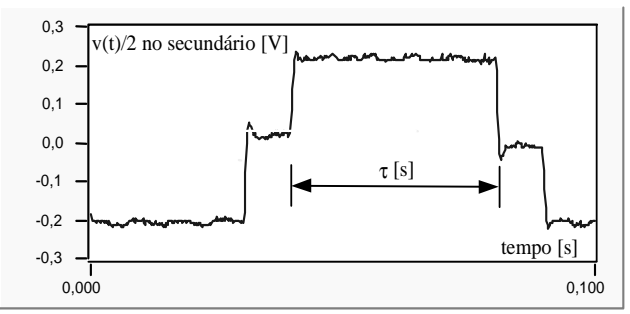

Figura 10: Forma de onda de tensão retangular secundário do quadro de Epstein, atenuada por um fator 2, $\mathrm{f}=10 \mathrm{~Hz}$.

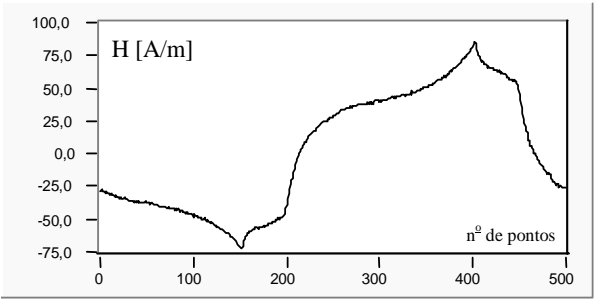

Figura 11: Forma de onda de $\mathrm{H}[\mathrm{A} / \mathrm{m}]$ relativa à figura 10.

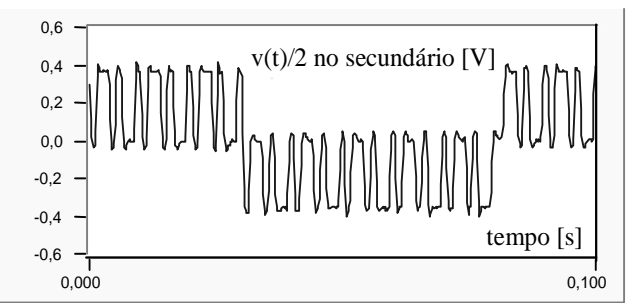

Figura 12: Forma de onda de tensão PWM no secundário do quadro de Epstein, atenuada por um fator 2, com $\mathrm{f}=10 \mathrm{~Hz}$. 


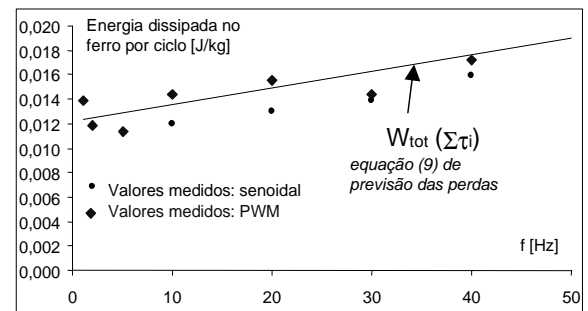

Figura 13: Energia dissipada: formas de onda de tensão no secundário senoidal e PWM $\left(\mathrm{B}_{m}=0,8 \mathrm{~T}\right.$ e $\left.\mathrm{F}_{c}=1\right)$.

\section{CONCLUSÃO}

Existem muitos parâmetros que influenciam as perdas magnéticas, tanto na sua natureza como no processo de medida. Quando se realiza a modelagem, a medição e a análise, deve-se atuar com critérios rigorosos quando da influência de parâmetros e fenômenos supostamente possíveis de serem negligenciados. Sabe-se que há o problema dos cantos do quadro de Epstein, tanto no que se refere a fenômenos magnéticos como em relação ao valor da massa efetiva de aço utilizada no valor quantitativo da perda medida. Estes fatores, e outros aqui não mencionados por sua complexidade ou por fugirem do escopo deste trabalho, não foram abordados neste artigo, os quais também podem gerar imprecisões. Na amostra de material utilizado, juntamente com outros fenômenos, eles podem ser as causas da não conformidade do modelo com os valores medidos na caracterização para induções superiores a 1,4T.

A contribuição deste trabalho é a metodologia utilizada para a determinação das perdas no ferro e o modo de alimentação do quadro de Epstein. A maneira de caracterização em função da variação da indução máxima proposta neste trabalho parece ser de grande efetividade em tornar evidente possíveis imprecisões, tanto de caráter experimental como nos modelos dos fenômenos em questão. A formulação para a estimação das perdas em aço silício proposta por Amar e Protat (1994) se mostra eficaz e simples, carecendo ainda de um modelo para perda por histerese.

\section{REFERÊNCIAS}

ACESITA Aços Especiais. "Aços ao Silício de Grão Orientado e Aços ao Silício de Grão Não Orientado".

Allano, S., A. Lebouc, R. Perret (1987). Pertes Dans les Circuits Magnétiques: une Modélisation Complexe. Revue Générale dÉlectricité, nº3, pp. 35-44.

Amar, M.; F. Protat (1994). A Simple Method for the
Estimation of Power Losses in Silicon Iron Sheets under Alternating Pulse Voltage Excitation. IEEE Transactions on Magnetics, Vol 30, $\mathrm{n}^{\circ}$ 2. pp. 942944.

Basak, A. \& C. R. G. Higgs(1982). Flux Distribution in Three Phase Transformer Cores With Various T-Joint Geometries, IEEE Transactions on Magnetics, vol. $18, \mathrm{n}^{\circ} 2$, pp. $670-673$.

Batistela, N.J., A. J. Perin (1995). A Fixed Frequency Sliding Mode Control For Voltage Souce Inverter. III Brazilian Power Electronics Conference - COBEP'95, pp 229-234.

Batistela, N.J., N. Sadowski, R. Carlson, J.V. Leite (2000). A Caracterização Magnética de Lâminas de Aço Silício e a Evolução das Perdas no Ferro Sob Vários Regimes de Indução. CBA Congresso Brasileiro de Automática, Florianópolis, pp. 961-966.

Bertotti, G. (1983). Space-Time Correlation Properties of the Magnetization Process and Eddy Current Losses: Theory. J. Appl. Phys., vol. 54, $\mathrm{n}^{o} 9$, pp. $5293-5305$.

Bertotti, G. (1984). Space-Time Correlation Properties of the Magnetization Process and Eddy Current Losses: Applications. I. Fine wall Spacing. J. Appl. Phys., vol.55, $\mathrm{n}^{\circ} 12$, pp. 4339-4355.

Bertotti, G. (1985). Physical Interpretation of Eddy Current Losses in Ferromagnetic Materials. I. Theoretical considerations. J. Appl. Phys., vol. 57, $\mathrm{n}^{\circ}$ 6, pp. 2110 - 2126.

Bertotti, G. (1988). General Properties of Power Losses in Soft Ferromagnetic Materials. IEEE Trans. Magn., vol. 24, $\mathrm{n}^{\circ}$ 1, 1988, pp. $621-630$.

Errard, Serge. (1993). Determination et Analyse des Pertes Supplementaires Developpees dans les Toles des Machines Alimentees par Convertisseur Statique. Tese de doutorado, L'Institut National Polytechnique de Grenoble.

Ferreira da Luz, M.V., N. Sadowski, R. Carlson, J.P.A. Bastos, N.J. Batistela (2000). Calculation of Iron Losses in Induction Motors using Finite Element Method". ICEM Finlândia, Artigo aceito.

Fiorillo, F., A. Novikov (1990). An Improved Approach to Power Losses in Magnetic Laminations under Nonsinusoidal Induction Waveform. IEEE Transactions on Magnetics, vol.26, nº 5 , pp 2904-2910. 
Haller, T. R. \& J.J. Kramer (1970). Observation of Dynamic Domain Size Variation in a Silicon-Iron Alloy. J. Appl. Phys., vol. 41, no 3, pp. $1034-1035$.

Hernandez, E.D.M., C.S. Muranaka, J.R. Cardoso (2000). Identification of the Jiles-Atherton Model Parameters Using Random and Deterministics Searches. Physica B, 275, pp. 212-215.

Landgraf, F. J. G., M. Emura, J.C. Teixeira, M.F. Campos, C.S. Muranaka (1999). Anisotropy of the Magnetic Losses Components in Semi-Processed Electrical Steels. Journal of Magnetism and Magnetic Materials,196-197, pp 380-381.

Landgraf, F. J. G., M. Emura, J.C. Teixeira, M.F. Campos (2000). Effect of Grain Size, Deformation, Aging and Anisotropy on Hysteresis Loss of Electrical Steels. Journal of Magnetism and Magnetic Materials,215-216, pp 97-99.

Liwschitz, M. Capítulo III. ÉDITIONS SPES LAUSANNE. Calcul des Machines Électriques, Tome I. pp 97 a 99. Bibliothèque de L'Ingénieur. Éditions Spes Lausanne, França.

Moses, A. J., G. H. Shirkoohi (1989). Importance of Harmonic Phase Angle in Prediction of Iron Loss Under Distorted Magnetization, Physica Scripta, vol.39, $\mathrm{n}^{\circ} 17$, pp. 523-525.

Nakata, T.; N. Takahashi, K. Fujiwara, M. Nakano, K. Matsubara. Physica (1989). Scripta, vol. 39, pp. 645 -647 . 